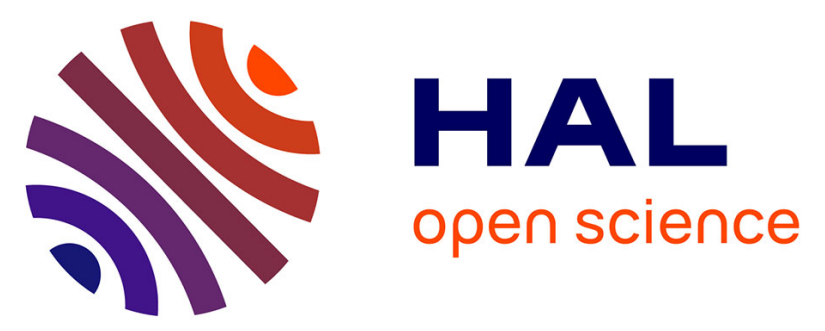

\title{
Solvation force and adsorption isotherm of a fluid mixture in nanopores of complex geometry based on fundamental measure theory
}

\author{
Q D Ha, Tien Dung Le, Irina Panfilov, Christian Moyne
}

\section{To cite this version:}

Q D Ha, Tien Dung Le, Irina Panfilov, Christian Moyne. Solvation force and adsorption isotherm of a fluid mixture in nanopores of complex geometry based on fundamental measure theory. Journal of Physics: Condensed Matter, 2021, 33 (33), pp.335002. 10.1088/1361-648X/ac0ab5 . hal-03476442

\section{HAL Id: hal-03476442 \\ https://hal.univ-lorraine.fr/hal-03476442}

Submitted on 12 Dec 2021

HAL is a multi-disciplinary open access archive for the deposit and dissemination of scientific research documents, whether they are published or not. The documents may come from teaching and research institutions in France or abroad, or from public or private research centers.
L'archive ouverte pluridisciplinaire HAL, est destinée au dépôt et à la diffusion de documents scientifiques de niveau recherche, publiés ou non, émanant des établissements d'enseignement et de recherche français ou étrangers, des laboratoires publics ou privés.

\section{(1) (1) $\$$}

Distributed under a Creative Commons Attribution - NonCommercial - NoDerivatives 44.0 


\title{
Solvation force and adsorption isotherm of a fluid mixture in nanopores of complex geometry based on Fundamental Measure Theory
}

\author{
Q. D. Ha ${ }^{1}$, T. D. Le ${ }^{1}$, I. Panfilov ${ }^{1}$, C. Moyne ${ }^{1}$ \\ ${ }^{1}$ University of Lorraine, CNRS, LEMTA, F-54000 Nancy, France
}

\begin{abstract}
A novel method based on the Fundamental Measure Theory (FMT) is developed to calculate the solvation force and adsorption isotherm of a Lennard-Jones fluid mixture in complex geometries. Fast Fourier Transform and 3D-voxel discretization are used for accurately computing the confined fluid densities in a closed pore of arbitrary geometry. Given the fluid densities, the solvation force distribution at the solid surface can be calculated using a new formulation from either mechanical or thermodynamic approach. Understanding the solvation force behavior, which depends on many factors such as pore geometry, confined density distribution, molecule size, is very important to analyze the pore deformation from a poromechanical point of view. Special attention in the numerical simulations is given to the adsorption problem of $\mathrm{CH}_{4}$ and $\mathrm{CO}_{2}$ gas mixture in ellipsoidal pore.
\end{abstract}

Keywords: Adsorption Isotherm, Gas Mixture Adsorption, Density Functional Theory, Fundamental Measure Theory, Solvation Force

\section{Introduction}

Gas adsorption phenomena in nanopores of size of several nanometers play a crucial role in many areas, in particular for unconventional gas reservoirs [1, 2]. Shale contains organic material, namely kerogen, composed of nanopores where a large quantity of methane is in sorbed state with a density much higher than in large pores [3,4]. In coalbed methane reservoir, the main gas quantity in coal seam is stored within the nanopore network in the coal matrix. Moreover, the

$\ddagger$ Corresponding author 
adsorbed gas pressure in the nanopore has a magnitude much larger than the bulk pressure in large pores $[5,6,7]$ (natural fractures, cleats, etc) which may induce swelling/shrinkage of the medium $[8,6,9,7]$. Understanding such complex phenomena becomes crucial to predict the mechanical behavior at the macroscale and the gas production curve. In this context, adsorption isotherm has attracted considerable attention in the literature. Some advanced theories as Molecular Dynamics, Monte Carlo simulations and Density Functional Theory [10] can be applied to capture the strongly inhomogeneous behavior of sorbed gas in order to compute the gas density profiles within the pore and the adsorption-induced pressure.

Within the framework of the Density Functional Theory [10], the Fundamental Measure Theory (FMT) was developed by Rosenfeld [11, 12] for a hard-sphere mixture fluid. The weighted densities, which are convolutions of the local densities with weight functions, are introduced to construct the excess free energy based on the Percus-Yevick relation (PY) [13] for the direct correlation function of a hard-sphere fluid. Further, to obtain a more precise model, the FMT model was slightly modified to verify the semi-empirical Mansoori-Carnahan-Starling-Leland (MCSL) relation for the fluid pressure[14]. This new version called "White-Bear" is well explained in a review by Roth [15]. The FMT approach is also relevant for confined molecular system with longrange molecular interaction in addition to short-range hard-sphere repulsion and with an external solid wall/fluid interaction obeying the Lennard-Jones potential [16].

Numerical simulations and applications of the FMT for a gas mixture adsorption problem are widely studied in the literature. Gonzalez et al. [17] applied the original FMT of Rosenfeld and the modified FMT of Tarazona [18] in cylindrical and spherical pores and compared these results with Monte Carlo simulations interpreting the differences between both theories. Gas mixture adsorption in the nanopores of organic material in unconventional reservoirs for slit and spherical geometries has been reported in $[3,9,7]$ considering a Lennard-Jones fluid. Bernet et al. [19], adding the tensorial weighted densities of Tarazona to improve convergence at high density, numerically investigated the three dimensional fluid distribution using the Fast Fourier Transform.

Given the local fluid densities in the nanopores, calculation of the adsorption-induced force exerted on the solid surface by the confined fluids, namely the solvation force, is important as this force can be much higher than the bulk fluid pressure with a considerable impact on the mechanical behavior at the macroscale. Ravikovitch et al. [5] dealt with the deformation of a matrix solid composed of spherical pores in which the solvation force was introduced through the 
derivative of the grand potential with respect to the pore radius. Le et al. [9, 7] studied the impact of the solvation force on the poromechanics and transport in coalbed methane containing spherical nanopores through homogenization approach. However, to the best of the authors' knowledge, no work has attempted to compute the solvation force at the solid wall in arbitrary geometries for a Lennard-Jones fluid. This paper aims at filling this gap.

First, a numerical method is developed to solve the FMT formulation, considering both the Lennard-Jones interaction between the molecules and the exterior potential due to the solid/fluid interaction, in order to compute the gas mixture density profiles in a generalized pore geometry in three dimensions. The pore domain is discretized by voxels of the same size. In this configuration, the convolution terms are computed in Fourier space giving a huge advantage in terms of time calculation and computer resources. The accuracy of the method depends strongly on the number of voxels. Given the fluid densities, the solvation force, which is the force exerted on the solid wall by the fluid phase, is then calculated. Two different approaches are proposed to obtain the formulation of the solvation force. Numerical simulations validate the approach by comparison with Monte Carlo simulations. As an example of more complex geometry, the oblate spheroidal pore is studied. In addition, special attention is given to the application to the enhanced coalbed methane recovery by $\mathrm{CO}_{2}$ injection. Numerical results show that the adsorption of $\mathrm{CO}_{2}$ in coal seams dominates the methane adsorption. Therefore, injected $\mathrm{CO}_{2}$ can replace $\mathrm{CH}_{4}$ in the nanopores and enhance the methane production. More, it is shown that the solvation force is negative with a magnitude much larger than the bulk pressure. Hence, this force must be taken into account for studying the mechanical properties at the macroscale [9, 7].

The paper is organized as follows. In section 2, the FMT approach for a hard-sphere mixture system considering a Lennard-Jones intermolecular interaction and an exterior potential is presented. In section 3, two different approaches, namely mechanics and thermodynamics approaches, are developed to calculate the solvation force. The numerical method is explained in section 4 and numerical simulations are performed in section 5. The conclusions are drawn in section 6 . 


\section{Local densities of a confined fluid mixture}

\subsection{Density Functional Theory}

In this section, the Density Functional Theory is briefly recalled to calculate the densities of inhomogeneous fluid mixture near a solid surface. The fluid mixture is composed of $\mathrm{N}$ species of number densities $\rho_{i}(1 \leq i \leq \mathrm{N})$. In the grand canonical ensemble, with fixed volume, temperature and chemical potential, the grand potential is minimum at equilibrium. The solid wall exerts a force on each molecule of component $i$ given by the external potential $V_{i}^{e x t}$. As the fluid densities $\rho_{i}$ are inhomogeneous depending on the position $\mathbf{r}$, a non-local formulation of the grand potential $\Omega$ is given by [20]

$$
\Omega\left[\left\{\rho_{i}(\mathbf{r})\right\}\right]=\mathscr{F}\left[\left\{\rho_{i}(\mathbf{r})\right\}\right]+\sum_{i} \int \rho_{i}(\mathbf{r})\left[V_{i}^{e x t}(\mathbf{r})-\mu_{i}\right] \mathrm{d} \mathbf{r}
$$

where $\mathscr{F}$ denotes the intrinsic free energy being a functional of $\rho_{i}, \mu_{i}=\psi_{i}+V_{i}^{\text {ext }}$ the total chemical potential of component $i$ which is sum of the intrinsic chemical potential $\psi_{i}$ and of the exterior potential $V_{i}^{\text {ext }}$.

The inhomogeneous fluid system is assumed to be in equilibrium with a large volume of an external fluid, namely bulk fluid. At equilibrium, the chemical potential is uniform, $\mu_{i}=\mu_{i}^{b}$ where $\mu_{i}^{b}$ represents the chemical potential of component $i$ in the bulk. The latter can be decomposed into an ideal part corresponding to perfect gas and an excess part $\mu_{i}^{b^{e x}}$ taking into account the molecule-molecule interaction

$$
\mu_{i}^{b}=k_{B} T \ln \left(p_{i}^{b} \Lambda_{i}^{3}\right)+\mu_{i}^{b^{e x}}
$$

where $k_{B}$ is the Boltzmann constant, $T$ the absolute temperature, $\Lambda_{i}$ the de Broglie thermal wavelength. At equilibrium, the minimization of the grand potential gives

$$
\frac{\delta \Omega\left[\left\{\rho_{i}\right\}\right]}{\delta \rho_{i}(\mathbf{r})}=0
$$

where $\delta / \delta$ denotes the functional derivative.

The intrinsic free energy $\mathscr{F}$ can be decomposed into an ideal part and an excess part

$$
\mathscr{F}=\mathscr{F}^{i d}+\mathscr{F}^{e x}
$$

The ideal part is given as

$$
\mathscr{F}^{i d}\left[\left\{\rho_{i}(\mathbf{r})\right\}\right]=k_{B} T \sum_{i} \int \rho_{i}(\mathbf{r})\left(\ln \left[\rho_{i}(\mathbf{r}) \Lambda_{i}^{3}\right]-1\right) \mathrm{d} \mathbf{r}
$$


The derivative of the intrinsic free energy with respect to $\rho_{i}(\mathbf{r})$ yields

$$
\frac{\delta \mathscr{F}\left[\left\{\rho_{i}\right\}\right]}{\delta \rho_{i}(\mathbf{r})}=k_{B} T \ln \left[\rho_{i}(\mathbf{r}) \Lambda_{i}^{3}\right]+\frac{\delta \mathscr{F}^{e x}\left[\left\{\rho_{i}\right\}\right]}{\delta \rho_{i}(\mathbf{r})}
$$

Inserting (1) into (3) and using (6) give

$$
k_{B} T \ln \left[\rho_{i}(\mathbf{r}) \Lambda_{i}^{3}\right]+\frac{\delta \mathscr{F}^{e x}\left[\left\{\rho_{i}\right\}\right]}{\delta \rho_{i}(\mathbf{r})}+V_{i}^{e x t}(\mathbf{r})-\mu_{i}=0
$$

The density of component $i$ can be deduced from the above relation by using (2)

$$
\rho_{i}(\mathbf{r})=\rho_{i}^{b} \exp \left[\beta\left(\mu_{i}^{b^{e x}}-\frac{\delta \mathscr{F}^{e x}\left[\left\{\rho_{i}\right\}\right]}{\delta \rho_{i}(\mathbf{r})}-V_{i}^{e x t}(\mathbf{r})\right)\right]
$$

where $\beta=1 /\left(k_{B} T\right)$.

Given the excess free energy $\mathscr{F}^{e x}$ and the exterior potential $V_{i}^{\text {ext }}$ for each component $i$, the local densities $\rho_{i}(\mathbf{r})$ can be computed from (8).

It is important to keep in mind that the fluid molecule $i$ is supposed to be a hard sphere of diameter $d_{i}$. Within the perturbation approach [16], the excess free energy can be decomposed into two components: a hard-sphere short-range repulsion and an attractive long-range intermolecular interaction component expressed in a mean field approximation for low density system

$$
\mathscr{F}^{e x}\left[\left\{\rho_{i}\right\}\right]=\mathscr{F}_{H S}^{e x}\left[\left\{\rho_{i}\right\}\right]+\sum_{i, j} \frac{1}{2} \iint \rho_{i}(\mathbf{r}) \rho_{j}\left(\mathbf{r}^{\prime}\right) w_{i j}\left(\mathbf{r}, \mathbf{r}^{\prime}\right) \operatorname{drd} \mathbf{r}^{\prime}
$$

where $w_{i j}\left(\mathbf{r}, \mathbf{r}^{\prime}\right)$ is the fluid-fluid interaction potential between a molecule $i$ at $\mathbf{r}$ and a molecule $j$ at $\mathbf{r}^{\prime}$.

The intermolecular potential $w_{i j}$ is represented by a 12-6 Lennard-Jones potential (LJ)

$$
w_{i j}\left(\mathbf{r}, \mathbf{r}^{\prime}\right)=w_{i j}(s)=4 \varepsilon_{i j}\left[\left(\frac{\sigma_{i j}}{s}\right)^{12}-\left(\frac{\sigma_{i j}}{s}\right)^{6}\right]
$$

where $s=\left|\mathbf{r}-\mathbf{r}^{\prime}\right|$ is the distance between molecules $i$ and $j . \varepsilon_{i j}$ and $\sigma_{i j}$ represent the energy and distance Lennard-Jones parameters related to a pair of molecules $i$ and $j . \varepsilon_{i j}$ and $\sigma_{i j}$ can be given from the single parameters for each component $\varepsilon_{i}$ and $\sigma_{i}$ as

$$
\varepsilon_{i j}=\sqrt{\varepsilon_{i} \varepsilon_{j}} \quad \text { and } \quad \sigma_{i j}=\frac{\sigma_{i}+\sigma_{j}}{2}
$$

The Weeks-Chandler-Anderson[21] (WCA) perturbation theory decomposes the LJ potential into a repulsive and an attractive potentials as

$$
w_{i j}^{r e p}(s)= \begin{cases}4 \varepsilon_{i j}\left[\left(\frac{\sigma_{i j}}{s}\right)^{12}-\left(\frac{\sigma_{i j}}{s}\right)^{6}\right]+\varepsilon_{i j} & s \leq 2^{1 / 6} \sigma_{i j} \\ 0 & s>2^{1 / 6} \sigma_{i j}\end{cases}
$$


and

$$
w_{i j}^{a t t}(s)=\left\{\begin{array}{lc}
-\varepsilon_{i j} & s \leq 2^{1 / 6} \sigma_{i j} \\
4 \varepsilon_{i j}\left[\left(\frac{\sigma_{i j}}{s}\right)^{12}-\left(\frac{\sigma_{i j}}{s}\right)^{6}\right] & s>2^{1 / 6} \sigma_{i j}
\end{array}\right.
$$

In this work, only the attractive part of the potential is considered to describe the fluid-fluid interaction potential $w_{i j}$ in (9) when the repulsive part is treated as a hard-sphere interaction. The excess free energy for this interaction is calculated from the Fundamental Measure Theory (FMT) which is discussed in the following subsection.

\subsection{Fundamental Measure Theory}

The Fundamental Measure Theory (FMT) has been developed by Rosenfeld [11] for modeling a hard-sphere mixture system based on weight functions and weighted densities.

2.2.1. Weight functions and weighted densities Let introduce the weight functions for the component $i$ composed of four scalar and two vector functions

$$
\left\{\begin{aligned}
\omega_{3}^{i}(\mathbf{r}) & =H\left(R_{i}-|\mathbf{r}|\right) \\
\omega_{2}^{i}(\mathbf{r}) & =\delta\left(R_{i}-|\mathbf{r}|\right) \\
\omega_{1}^{i}(\mathbf{r}) & =\frac{\omega_{2}^{i}(\mathbf{r})}{4 \pi R_{i}} \\
\omega_{0}^{i}(\mathbf{r}) & =\frac{\omega_{2}^{i}(\mathbf{r})}{4 \pi R_{i}^{2}} \\
\omega_{2}^{i}(\mathbf{r}) & =\delta\left(R_{i}-|\mathbf{r}|\right) \frac{\mathbf{r}}{|\mathbf{r}|} \\
\omega_{1}^{i}(\mathbf{r}) & =\frac{\omega_{2}^{i}(\mathbf{r})}{4 \pi R_{i}}
\end{aligned}\right.
$$

where $H$ is the Heaviside function, $\delta$ the Dirac distribution and $R_{i}$ the radius of molecule $i$. It should be noted that the spatial integration of the four scalar weight functions $\omega_{\alpha}^{i}(\alpha=3,2,1,0)$ leads to the volume $V_{i}$, the surface $S_{i}$, the radius $R_{i}$ of the sphere $i$ and 1 respectively.

The weighted densities $n_{\alpha}(\mathbf{r})$ are defined as the convolution of the densities $\rho_{i}(\mathbf{r})$ and the weight functions $\omega_{i}(\mathbf{r})$ :

$$
n_{\alpha}(\mathbf{r})=\sum_{i}\left(\rho_{i} \otimes \omega_{\alpha}^{i}\right)(\mathbf{r})=\sum_{i} \int \mathrm{d} \mathbf{r}^{\prime} \rho_{i}\left(\mathbf{r}-\mathbf{r}^{\prime}\right) \omega_{\alpha}^{i}\left(\mathbf{r}^{\prime}\right)=\sum_{i} \int \mathrm{d} \mathbf{r}^{\prime} \rho_{i}\left(\mathbf{r}^{\prime}\right) \omega_{\alpha}^{i}\left(\mathbf{r}-\mathbf{r}^{\prime} \gamma 15\right)
$$

where the symbol $\otimes$ represents the convolution operator in the three-dimensional space. Here, $\alpha$ refers to four scalar and two vector weighted densities corresponding to their weight functions. In 
a bulk fluid with a uniform density $\rho_{i}^{b}$, the two vector weighted densities $\mathbf{n}_{\mathbf{1}}$ and $\mathbf{n}_{\mathbf{2}}$ vanish whereas the four scalar ones converge to the Scaled-Particle-Theory (SPT) variables [20] given as

$$
\begin{aligned}
n_{3}^{b} & =\frac{4 \pi}{3} \sum_{i} \rho_{i}^{b} R_{i}^{3}=\sum_{i} \rho_{i}^{b} V_{i} \\
n_{2}^{b} & =4 \pi \sum_{i} \rho_{i}^{b} R_{i}^{2}=\sum_{i} \rho_{i}^{b} S_{i} \\
n_{1}^{b} & =\sum_{i} \rho_{i}^{b} R_{i} \\
n_{0}^{b} & =\sum_{i} \rho_{i}^{b}
\end{aligned}
$$

2.2.2. Excess free energy of a hard-sphere mixture The excess free energy of a hard-sphere mixture can be presented as $[11,12]$

$$
\beta \mathscr{F}_{H S}^{e x}\left[\left\{\rho_{i}(\mathbf{r})\right\}\right]=\int \Phi\left[n_{\alpha}\left(\mathbf{r}^{\prime}\right)\right] \mathrm{d} \mathbf{r}^{\prime}
$$

where the reduced free energy per unit volume $\Phi$ is function of the weighted densities. From the deconvolution of the Mayer- $f$ function, Rosenfeld has developed an ansatz for $\Phi$

$$
\Phi=-n_{0} \ln \left(1-n_{3}\right)+\frac{n_{1} n_{2}-\mathbf{n}_{\mathbf{1}} \mathbf{n}_{\mathbf{2}}}{1-n_{3}}+\frac{n_{2}^{3}-3 n_{2} \mathbf{n}_{\mathbf{2}} \mathbf{n}_{\mathbf{2}}}{24 \pi\left(1-n_{3}\right)^{2}}
$$

In the bulk, the vectors $\mathbf{n}_{1}$ and $\mathbf{n}_{2}$ vanish so that we have

$$
\Phi_{b}=-n_{0} \ln \left(1-n_{3}\right)+\frac{n_{1} n_{2}}{1-n_{3}}+\frac{n_{2}^{3}}{24 \pi\left(1-n_{3}\right)^{2}}
$$

The derivative of the excess free energy with respect to the density $\rho_{i}$ is obtained as

$$
\beta \frac{\delta \mathscr{F}_{H S}^{e x}\left[\left\{\rho_{i}\right\}\right]}{\delta \rho_{i}(\mathbf{r})}=\sum_{\alpha} \int d \mathbf{r}^{\prime} \frac{\partial \Phi}{\partial n_{\alpha}}\left(\mathbf{r}^{\prime}\right) \frac{\delta n_{\alpha}\left(\mathbf{r}^{\prime}\right)}{\delta \rho_{i}(\mathbf{r})}
$$

From the definition (15), the derivative of the weighted functions with respect to the density is given as

$$
\frac{\delta n_{\alpha}\left(\mathbf{r}^{\prime}\right)}{\delta \rho_{i}(\mathbf{r})}=\int d \mathbf{r}^{\prime \prime} \frac{\delta \rho_{i}\left(\mathbf{r}^{\prime \prime}\right)}{\delta \rho_{i}(\mathbf{r})} \omega_{\alpha}^{i}\left(\mathbf{r}^{\prime}-\mathbf{r}^{\prime \prime}\right)=\omega_{\alpha}^{i}\left(\mathbf{r}^{\prime}-\mathbf{r}\right)
$$

leading to

$$
\beta \frac{\delta \mathscr{F}_{H S}^{e x}\left[\left\{\rho_{i}\right\}\right]}{\delta \rho_{i}(\mathbf{r})}=\sum_{\alpha} \int d \mathbf{r}^{\prime} \frac{\partial \Phi}{\partial n_{\alpha}}\left(\mathbf{r}^{\prime}\right) \omega_{\alpha}^{i}\left(\mathbf{r}^{\prime}-\mathbf{r}\right)
$$

Noting that $\omega_{\alpha}^{i}\left(\mathbf{r}-\mathbf{r}^{\prime}\right)=\omega_{\alpha}^{i}\left(\mathbf{r}^{\prime}-\mathbf{r}\right)$ for the scalar weight functions whereas for the vector ones $\boldsymbol{\omega}_{\alpha}^{i}\left(\mathbf{r}-\mathbf{r}^{\prime}\right)=-\boldsymbol{\omega}_{\alpha}^{i}\left(\mathbf{r}^{\prime}-\mathbf{r}\right)$, the above equation becomes

$$
\begin{aligned}
\beta \frac{\delta \mathscr{F}_{H S}^{e x}\left[\rho_{i}\right]}{\delta \rho_{i}(\mathbf{r})} & =\sum_{\alpha=0}^{3} \int d \mathbf{r}^{\prime} \frac{\partial \Phi}{\partial n_{\alpha}}\left(\mathbf{r}^{\prime}\right) \omega_{\alpha}^{i}\left(\mathbf{r}-\mathbf{r}^{\prime}\right)-\sum_{\alpha=1}^{2} \int d \mathbf{r}^{\prime} \frac{\partial \Phi}{\partial \mathbf{n}_{\alpha}}\left(\mathbf{r}^{\prime}\right) \omega_{\alpha}^{i}\left(\mathbf{r}-\mathbf{r}^{\prime}\right) \\
& =\sum_{\alpha=0}^{3} \frac{\partial \Phi}{\partial n_{\alpha}} \otimes \omega_{\alpha}^{i}-\sum_{\alpha=1}^{2} \frac{\partial \Phi}{\partial \mathbf{n}_{\alpha}} \otimes \omega_{\alpha}^{i}
\end{aligned}
$$


The derivatives of the reduced free energy with respect to the weighted functions are easily obtained from (18). On the other hand, the excess chemical potential of the bulk is the sum of the excess chemical potential due to hard-sphere interaction and of the Van der Walls interactions between molecules. It is given by

$$
\begin{aligned}
\beta \mu_{i}^{b^{e x}} & =\beta\left(\mu_{i}^{b^{e x}}\right)_{\mathrm{HS}}+\beta\left(\mu_{i}^{b^{e x}}\right)_{\mathrm{VdW}}=\sum_{\alpha=0}^{3} \frac{\partial \Phi_{b}}{\partial n_{\alpha}^{b}} \frac{\partial n_{\alpha}^{b}}{\partial \rho_{i}^{b}}+\beta \sum_{j} \int_{|\mathbf{r}|>d_{i j}} \rho_{j}^{b} w_{i j}(|\mathbf{r}|) \mathrm{d} \mathbf{r} \\
& =\frac{\partial \Phi_{b}}{\partial n_{3}^{b}} V_{i}+\frac{\partial \Phi_{b}}{\partial n_{2}^{b}} A_{i}+\frac{\partial \Phi_{b}}{\partial n_{1}^{b}} R_{i}+\frac{\partial \Phi_{b}}{\partial n_{0}^{b}}+\beta \sum_{j} \int_{|\mathbf{r}|>d_{i j}} \rho_{j}^{b} w_{i j}(|\mathbf{r}|) \mathrm{d} \mathbf{r}
\end{aligned}
$$

where the mean diameter is $d_{i j}=\left(d_{i}+d_{j}\right) / 2$.

Given the exterior potential $V_{i}^{e x t}$, the local densities of the fluid mixture can be calculated by relation (8) making use of the preceding result (FMT) for the excess free energy contribution of the hard-sphere fluid.

\subsection{Exterior potential}

The exterior potential $V_{i}^{\text {ext }}(\mathbf{r})$, which represents the interaction between the solid atoms at the pore surface and a molecule $i$ at position $\mathbf{r}$, can be calculated by a surface integral of the LennardJones potential 12-6, $v_{i}$, between the molecule $i$ and the solid surface. It strongly depends on the geometrical form of the solid surface. Only the first atomic layer of the solid is supposed to interact with the gas molecules.

Let consider a solid surface $S$ of a pore of volume $V$ containing the gas mixture of $\mathrm{N}$ components. Each atom of the solid surface element $\mathrm{d} S$ exerts on the molecules of fluid component $i$ occupying the volume element $\mathrm{d} V$ a potential $v_{i}(s)$ where $s$ is the distance between $\mathrm{d} S$ and $\mathrm{d} V$ defined by

$$
s^{2}=\left(x-x_{S}\right)^{2}+\left(y-y_{S}\right)^{2}+\left(z-z_{S}\right)^{2}
$$

where $\mathbf{r}=(x, y, z)$ denotes the Cartesian coordinates in the fluid volume $V$ whereas $\mathbf{r}_{s}=\left(x_{S}, y_{S}, z_{S}\right)$ designates the Cartesian coordinates of the surface $S . v_{i}(s)$ can be defined as a 12-6 Lennard-Jones potential-type. Let $\varepsilon_{s}$ and $\sigma_{s}$ be the Lennard-Jones parameters of the solid atom, $\rho_{s}$ and $\Delta$ the solid surface density and the distance between two solid layers at the solid surface. The pair solid/fluid Lennard-Jones parameters are given as

$$
\varepsilon_{s i}=\sqrt{\varepsilon_{s} \varepsilon_{i}} \quad \text { and } \quad \sigma_{s i}=\frac{\sigma_{s}+\sigma_{i}}{2}
$$


The interaction potential $v_{i}(s)$ is defined as

$$
v_{i}(s)=4 \varepsilon_{s i} \rho_{s} \Delta\left[\left(\frac{\sigma_{s i}}{s}\right)^{12}-\left(\frac{\sigma_{s i}}{s}\right)^{6}\right]
$$

The exterior potential $V_{i}^{e x t}$ exerted by the surface $S$ on the fluid component $i$ at the position $(x, y, z)$ is obtained by integrating the relation (27) over the surface $S$

$$
V_{i}^{e x t}(x, y, z)=\int_{S} 4 \varepsilon_{s i} \rho_{s} \Delta\left[\left(\frac{\sigma_{s i}}{s}\right)^{12}-\left(\frac{\sigma_{s i}}{s}\right)^{6}\right] \mathrm{d} S
$$

Applications for spherical and ellipsoidal geometries are carried out in Appendix A.

\section{Solvation force}

The solvation force is the force exerted on the solid wall by the confined fluid close to the wall. The main objective of this section is to develop a mathematical model to calculate precisely this force acting on any geometrical solid surface. Since the local fluid densities are known, two different approaches are developed in this work to compute the solvation force as described below.

\subsection{Mechanical approach}

The force exerted on a volume element $\mathrm{d} V$ of the fluid mixture at $\mathbf{r}$ by a solid surface element $\mathrm{d} S$ at $\mathbf{r}_{s}$ can be written as

$$
\mathrm{d} \mathbf{f}_{S \rightarrow f} \mathrm{~d} V=-\sum_{i} \rho_{i}(\mathbf{r}) \nabla v_{i}(s) \mathrm{d} S \mathrm{~d} V
$$

where $\mathrm{d} \mathbf{f}_{S \rightarrow f}$ is the force per unit volume of the fluid $\left(\mathrm{N} \mathrm{m}^{-3}\right)$ exerted by the surface element $\mathrm{d} S$ on the volume element $\mathrm{d} V . s$ defined in (25) is the distance between the elements $\mathrm{d} S$ and $\mathrm{d} V, v_{i}(s)$ the solid/fluid interaction potential.

By using the action-reaction principle, the force exerted on the surface element $\mathrm{d} S$ by the fluid element $\mathrm{d} V$ is

$$
\begin{aligned}
\mathrm{d} \mathbf{f}_{f \rightarrow s} \mathrm{~d} S & =-\mathrm{d} \mathbf{f}_{s \rightarrow f} \mathrm{~d} V=\sum_{i} \rho_{i}(\mathbf{r}) \nabla v_{i}(s) \mathrm{d} S \mathrm{~d} V \\
\mathrm{~d} \mathbf{f}_{f \rightarrow s} & =\sum_{i} \rho_{i}(\mathbf{r}) \nabla v_{i}(s) \mathrm{d} V=\sum_{i} \rho_{i}(\mathbf{r}) \frac{\mathrm{d} v_{i}(s)}{\mathrm{d} s} \nabla s \mathrm{~d} V
\end{aligned}
$$

where $\mathrm{d} \mathbf{f}_{f \rightarrow s}$ is the force per unit surface (stress) of the solid $\left(\mathrm{N} \mathrm{m}^{-2}\right)$ exerted on the surface element $\mathrm{d} S$ by the volume element $\mathrm{d} V$. 
From (25), the gradient of $s$ relative to $\mathbf{r}=(x, y, z)$ is given by $\nabla s=\left(\mathbf{r}-\mathbf{r}_{s}\right) / s$. Integrating (30) over the fluid volume gives the force exerted at point $\mathbf{r}_{s}$ on the solid surface

$$
\mathbf{f}_{f \rightarrow s}=-\sum_{i} \int_{V} \rho_{i}(\mathbf{r}) \frac{v_{i}^{\prime}(s)}{s}\left(\mathbf{r}_{S}-\mathbf{r}\right) \mathrm{d} \mathbf{r}
$$

where $v_{i}^{\prime}(s)=\mathrm{d} v_{i}(s) / \mathrm{d} s$.

It should be noted that the force acting on the surface $S$ is not necessary to be normal to the surface but contains a tangential component. We adopt the decomposition

$$
\mathbf{f}_{f \rightarrow s}=\mathbf{f}_{f \rightarrow s}^{n}+\mathbf{f}_{f \rightarrow s}^{\tau}
$$

where $\mathbf{f}_{f \rightarrow s}^{n}$ and $\mathbf{f}_{f \rightarrow s}^{\tau}$ refer to the normal and tangential components of the total force.

The solvation force $\Pi$ is defined as the force acting on the solid surface subtracting the bulk pressure $P_{b}[9,5]$. It yields

$$
\Pi=\mathbf{f}_{f \rightarrow s}-P_{b} \mathbf{n}
$$

where $\mathbf{n}$ denotes the unit vector directed outward to the fluid. The normal component of the solvation force acting on the solid surface, which swells or shrinks the pore, is given by

$$
\Pi^{n}=\mathbf{f}_{f \rightarrow s} \cdot \mathbf{n}-P_{b}
$$

whereas the magnitude of the tangential component is given by

$$
\Pi^{\tau}=\left\|\mathbf{f}_{f \rightarrow s}^{\tau}\right\|=\left\|\mathbf{f}_{f \rightarrow s}-\left(\mathbf{f}_{f \rightarrow s} \cdot \mathbf{n}\right) \mathbf{n}\right\|
$$

\subsection{Thermodynamic approach}

The external force exerted on the cavity surface of unit normal vector $\mathbf{n}$ directed outwards is a force per unit area $\sigma \cdot \mathbf{n}$, where $\boldsymbol{\sigma}$ is a symmetrical stress tensor. Let $\mathbf{u}$ be the small displacement at the solid surface. The work of the external force on the cavity is written as

$$
\delta \Omega=\int(\sigma \cdot \mathbf{n}) \cdot \mathrm{d} \mathbf{u} \mathrm{d} S
$$

leading to

$$
\left.\frac{\partial \Omega}{\partial \mathbf{u}}\right|_{T, \mu}=\int_{S} \sigma \cdot \mathbf{n} \mathrm{d} S
$$

Moreover, the definition of the grand potential (1) gives

$$
\left.\frac{\partial \Omega}{\partial \mathbf{u}}\right|_{T, \mu}=\left.\sum_{i} \int_{V} \rho_{i}(\mathbf{r}) \mathrm{d} \mathbf{r} \frac{\partial\left[V_{i}^{\text {ext }}(\mathbf{r} ; \mathbf{u})-\mu_{i}\right]}{\partial \mathbf{u}}\right|_{T, \mu}=\left.\sum_{i} \int_{V} \rho_{i}(\mathbf{r}) \mathrm{d} \mathbf{r} \frac{\partial V_{i}^{\text {ext }}(\mathbf{r} ; \mathbf{u})}{\partial \mathbf{u}}\right|_{T, \mu}
$$


The derivative with respect to $\mathbf{u}$ of the exterior potential $V_{i}^{\text {ext }}$ is given by

$$
\left.\frac{\partial V_{i}^{\text {ext }}(\mathbf{r} ; \mathbf{u})}{\partial \mathbf{u}}\right|_{T, \mu}=\frac{\mathrm{d}}{\mathrm{d} \mathbf{u}}\left[\int_{S} v_{i}(s) \mathrm{d} S\right]=\int_{S} v_{i}^{\prime}(s) \frac{\mathrm{d} s}{\mathrm{~d} \mathbf{u}} \mathrm{d} S
$$

where

$$
\begin{aligned}
s^{2}(\mathbf{u}) & =\left\|\left(\mathbf{r}_{S}+\mathbf{u}-\mathbf{r}\right)\right\|^{2} \\
\left.2 s \frac{\mathrm{d} s}{\mathrm{~d} \mathbf{u}}\right|_{\mathbf{u}=0} & =2\left(\mathbf{r}_{S}-\mathbf{r}\right)
\end{aligned}
$$

Finally, we have

$$
\begin{aligned}
\int_{S} \boldsymbol{\sigma} \cdot \mathbf{n} \mathrm{d} S & =\sum_{i} \int_{V} \rho_{i}(\mathbf{r}) \mathrm{d} \mathbf{r} \int_{S} \frac{v_{i}^{\prime}(s)}{s}\left(\mathbf{r}_{S}-\mathbf{r}\right) \mathrm{d} S \\
& =\sum_{i} \int_{S} \int_{V} \rho_{i}(\mathbf{r}) \mathrm{d} \mathbf{r} \frac{v_{i}^{\prime}(s)}{s}\left(\mathbf{r}_{S}-\mathbf{r}\right) \mathrm{d} S
\end{aligned}
$$

Identifying the two members of the preceding equation gives the force exerted by the fluid on the solid per unit area as

$$
\mathbf{f}_{f \rightarrow s}=-\boldsymbol{\sigma} \cdot \mathbf{n}=-\sum_{i} \int_{V} \rho_{i}(\mathbf{r}) \mathrm{d} \mathbf{r} \frac{v_{i}^{\prime}(s)}{s}\left(\mathbf{r}_{S}-\mathbf{r}\right)
$$

which recovers the result obtained by the mechanical approach (31).

\section{Numerical method}

In this section, a novel numerical method to accurately solve the system of equations of the FMT in any pore geometry is developed.

\subsection{Discretization}

A voxel-discretization of the 3D pore-structure is chosen to describe the domain. The porestructure is put into a cube of dimension $L \times L \times L$ and discretized into a 3D image composed of $N \times N \times N$ voxels as illustrated in figure 1 . The part outside of the pore domain is considered as a solid phase where all variables are set to zero. This discretization depends only on the number of voxels to capture the pore-structure information, in particular the pore surface. It should be kept in mind that the pore surface location is of utmost importance for the numerical simulations of the exterior potential and the solvation force. As a consequence, the number of voxels should be high enough to be capable of locating precisely the solid surface.

Given the position of the voxels located at the solid surface, in order to increase the accuracy, the solid surface is meshed with, for example, a Delaunay triangulation as illustrated on figure 2. 


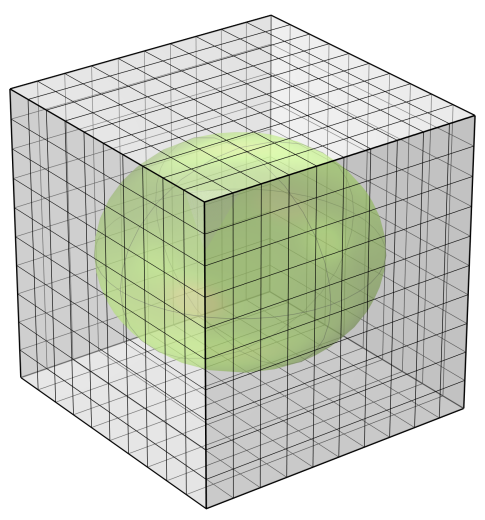

Figure 1: Example of a 3D voxel-discretization

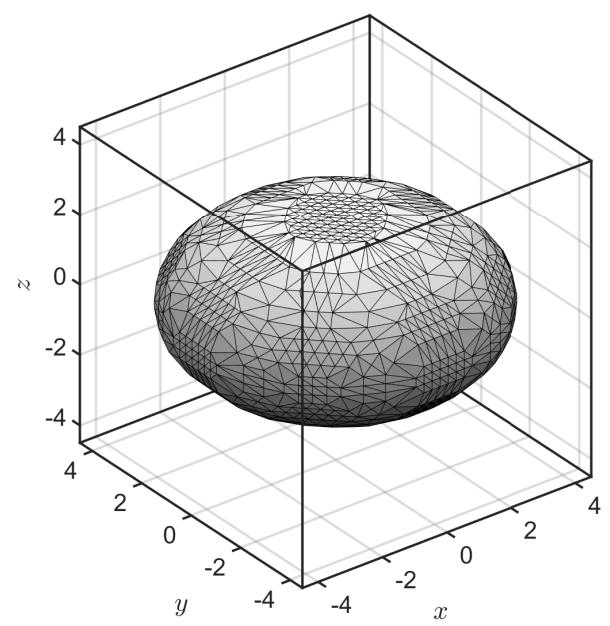

Figure 2: Surface mesh based on the voxel locations at the solid surface

\subsection{Fast Fourier Transform}

Since spatial convolutions in real space are transformed into simple products in Fourier space, calculating the hard-sphere excess free energy is an easier task in Fourier space. Moreover the weight functions in Fourier space are analytic, depending only on the Fourier parameter $k$ and the molecule radii $R_{i}$ (see Appendix B). In addition, as the weight functions are stiff (Dirac delta distribution for example), convolutions in real space require cumbersome interpolations. Working in Fourier space is therefore more accurate and efficient.

3D Fast Fourier Transforms are numerically carried out for densities and derivatives of the 
hard-sphere excess free energy with a FFT algorithm (fftn function in Matlab software). The calculation is performed in the full domain where densities are set to zero in the solid phase. On the other hand, the derivative of the long-range interaction component of the excess free energy with respect to densities is also a convolution, which can be computed in the Fourier space. It must be emphasized that the accuracy of this numerical method depends strongly on the number of voxels and on the solid surface description.

\section{Numerical simulation results}

\subsection{Validation of the numerical method}

First the numerical method is validated by comparison with Monte-Carlo simulations in different configurations.

5.1.1. One component hard-sphere fluid In the case of one component hard sphere of diameter $d$, numerical simulations are performed for a cylindrical pore in order to compare with Monte-Carlo simulations. A cylindrical cavity of radius $R_{p}=4 d$ and of finite length $L$ is contained in a box of dimension $L \times L \times L$ with $L=2 R_{p}$. In addition, the number of voxels used in the simulations is $N^{3}=512^{3}$. The $1 \mathrm{D}$ radial profile is compared with Monte-Carlo simulations reported in Bratko et al. [22] in figure 3. A very good agreement between the two methods is observed, which validates the proposed method.

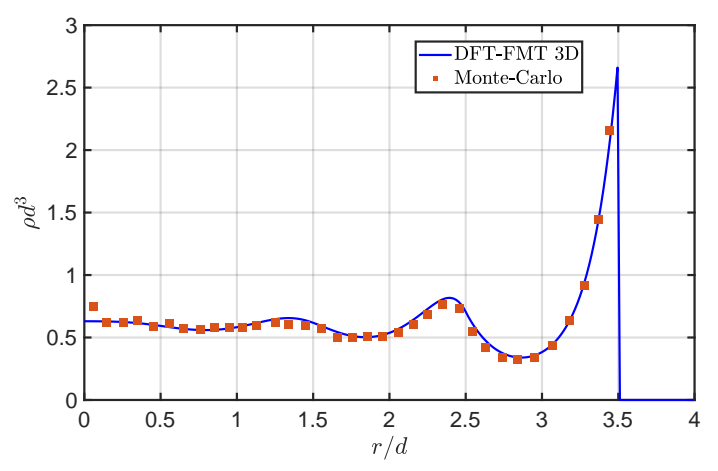

Figure 3: Radial density profile of a single component absorbed in a cylindrical pore of radius $R_{p}=4 d, \rho_{b} d^{3}=0.6$. 
5.1.2. Binary fluid mixture of different molecule diameters The reliability of the current method in the more complex case of a binary fluid mixture with different molecule diameters $d_{i}(i=s, b$ denoting small and big molecules in the mixture) is verified by comparison with a Monte-Carlo simulation reported in Kim et al. [23] in a spherical cavity of radius $R_{p}=5 d_{s}$ with $d_{b}=1.5 d_{s}$ and a bulk packing fraction $\eta=\pi\left(\rho_{s} d_{s}^{3}+\rho_{b} d_{b}^{3}\right) / 6=0.293$. The molecule numbers are fixed with $N_{s}=23$ and $N_{b}=66$ for the small and big molecules respectively. To do this, the corresponding bulk density $\rho_{b}$ is adjusted, keeping in mind that the FMT approach is based on the grand canonical ensemble specified by constant chemical potential, volume and temperature. Figure 4 displays the density profiles showing a very good agreement between the two approaches, validating the proposed model again.

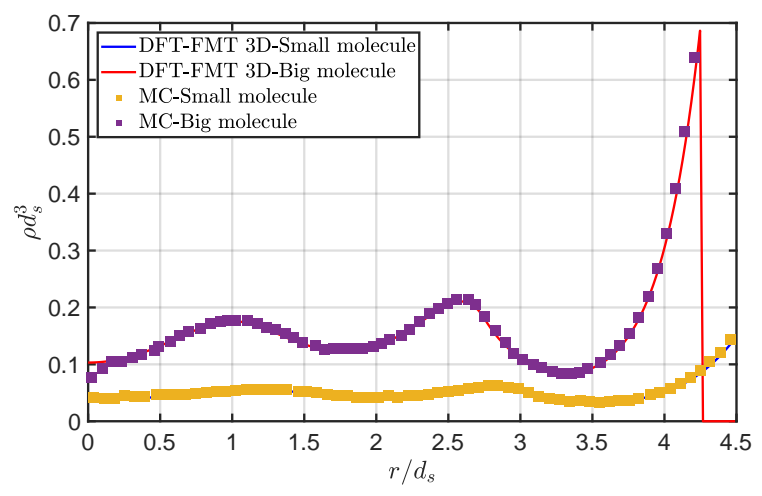

Figure 4: Density profiles of a binary fluid mixture in a spherical cavity of radius $R_{p}=5 d_{s}$. The particles numbers in the pore are fixed at $N_{s}=23$ and $N_{b}=66$. The diameter ratio between the two components is $d_{b} / d_{s}=1.5$. Numerical results are obtained by the $3 \mathrm{D}$-simulations based on our approach (continuous curves) and Monte-Carlo simulations reported in Kim et al.[23] (dotted curves).

\subsection{Adsorption isotherm of a gas mixture in a pore of complex geometry}

The adsorption isotherm of a Lennard-Jones fluid mixture in a pore of complex geometry is analyzed. Particular attention is paid to the adsorption competition between methane and carbon dioxide in enhanced coalbed methane recovery technique based on carbon dioxide injection. We consider an oblate spheroidal pore characterized by two semi-major axes $a=b$ and one semiminor axis $c$ with $a>c$. Let $d_{\mathrm{CH}_{4}}$ and $d_{\mathrm{CO}_{2}}$ be the diameters of $\mathrm{CH}_{4}$ and $\mathrm{CO}_{2}$ respectively. The 
pore size is chosen as $a=b=4.5 d_{\mathrm{CH}_{4}}$ and $c=3.5 d_{\mathrm{CH}_{4}}$ together with the normalized bulk densities $\rho_{\mathrm{CH}_{4}}^{b} d_{\mathrm{CH}_{4}}^{3}=\rho_{\mathrm{CO}_{2}}^{b} d_{\mathrm{CH}_{4}}^{3}=0.05$. The Lennard-Jones parameters used in the simulations are given in table 1.

\begin{tabular}{|c|c|c|c|}
\hline Parameter & Unit & $\mathrm{CH}_{4}[24,25]$ & $\mathrm{CO}_{2}[24,26]$ \\
\hline$\sigma_{s}$ & $\mathrm{~m}$ & $3.4 \times 10^{-10}$ & $3.4 \times 10^{-10}$ \\
$\varepsilon_{s i} / k_{B}$ & $\mathrm{~K}$ & 64 & 83 \\
$\Delta$ & $\mathrm{m}$ & $3.6 \times 10^{-10}$ & $3.6 \times 10^{-10}$ \\
$\rho_{s}$ & $\mathrm{~m}^{-3}$ & $114 \times 10^{27}$ & $114 \times 10^{27}$ \\
$\varepsilon_{i i} / k_{B}$ & $\mathrm{~K}$ & 148.2 & 246.15 \\
$\sigma_{i i}$ & $\mathrm{~m}$ & $3.75 \times 10^{-10}$ & $3.95 \times 10^{-10}$ \\
$T$ & $\mathrm{~K}$ & 333 & 333 \\
$d_{i}$ & $\mathrm{~m}$ & $3.53 \times 10^{-10}$ & $3.83 \times 10^{-10}$ \\
\hline
\end{tabular}

Table 1: Lennard-Jones parameters used in the simulation

Figure 5 displays the normalized densities of $\mathrm{CH}_{4}$ and $\mathrm{CO}_{2}$ in three-dimensional space. The gas density distributions are presented in two cutting planes $\mathrm{O} y z$ and $\mathrm{O} x z$ (where $\mathrm{O} x$ and $\mathrm{O} y$ refer to two semi-major axes, $\mathrm{O} z$ the semi-minor one) showing an adsorption peak in the vicinity of the solid surface (red) and the exclusion zone (blue) due to the hard-sphere/hard-solid interaction and the solid-fluid Lennard-Jones repulsion. Moreover, the $\mathrm{CO}_{2}$ density is much higher than the $\mathrm{CH}_{4}$ density with the same bulk density due to its higher adsorption potential.

To better analyze these phenomena, one-dimensional profiles are plotted in figures 6 according to the $\mathrm{O} x$ and $\mathrm{O} y$ axes respectively. The adsorption peaks and a much higher adsorption capacity of $\mathrm{CO}_{2}$ can be observed. The most important peaks are located in the vicinity of the wall and the $\mathrm{CO}_{2}$ peak can be 5 times higher than the $\mathrm{CH}_{4}$ one. On the other hand, it is important to note that geometries such as sphere or ellipsoid with analytic equations for their surfaces allow computing analytically the exterior potential. In order to generalize the method to an arbitrary pore, the pore surface should be identified and meshed as mentioned in section 4.1. Figures 6 show a comparison between results obtained by the analytic formula and Delaunay triangulation showing a very good agreement. 


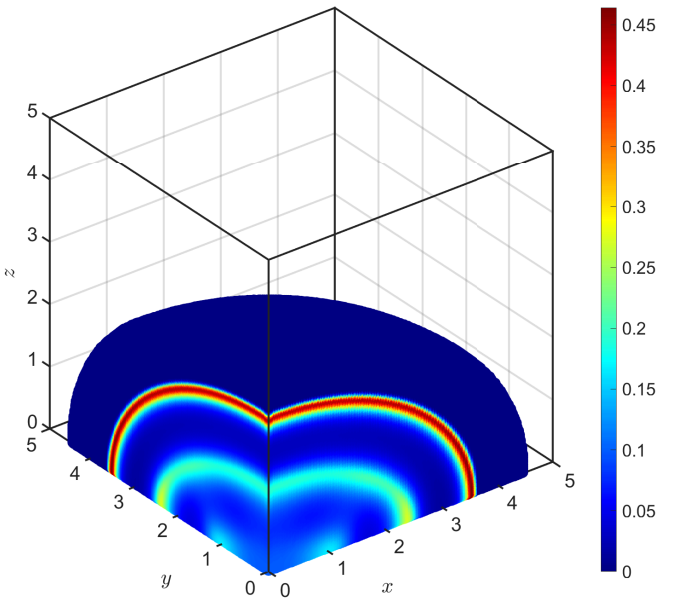

(a)

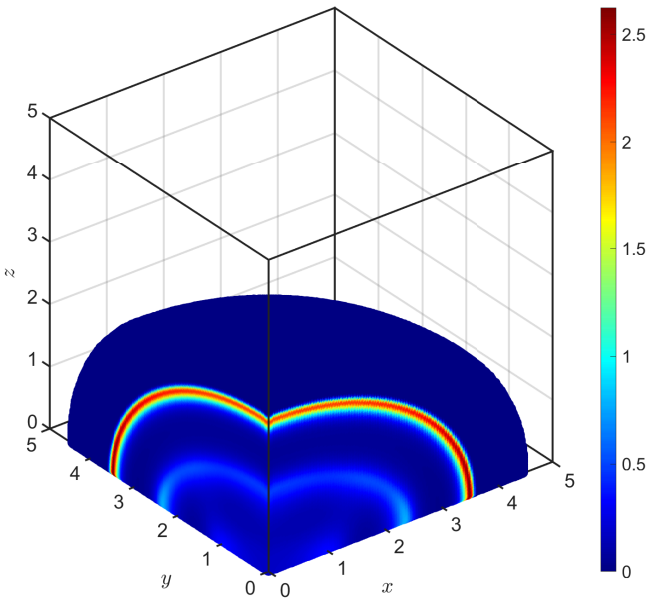

(b)

Figure 5: $3 \mathrm{D}$ view of (a) $\mathrm{CH}_{4}$ density and (b) $\mathrm{CO}_{2}$ density in an oblate spheroid

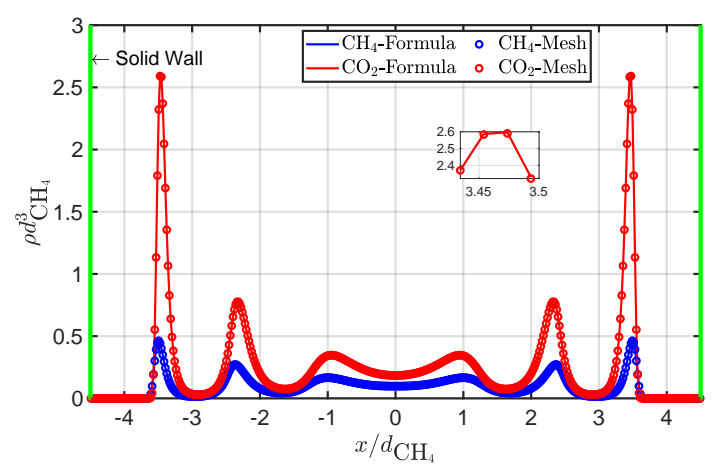

(a)

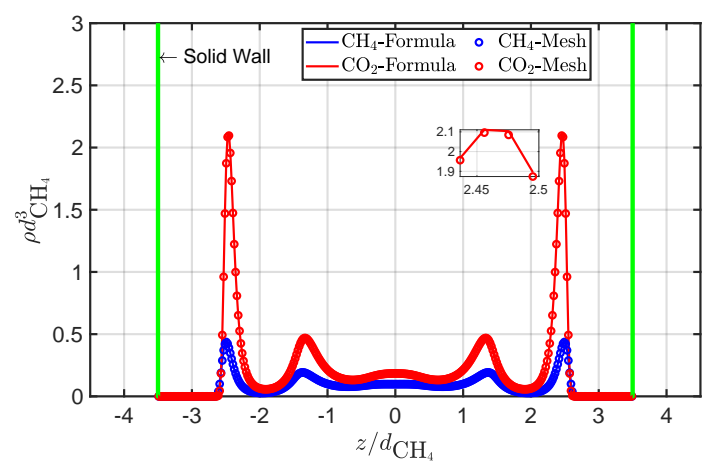

(b)

Figure 6: Gas density profiles in an oblate spheroid according to (a) $\mathrm{O} x$ axis and (b) $\mathrm{O} z$ axis for $a=b=4.5 d_{\mathrm{CH}_{4}}, c=3.5 d_{\mathrm{CH}_{4}}$ and the bulk densities $\rho_{\mathrm{CH}_{4}}^{b} d_{\mathrm{CH}_{4}}^{3}=\rho_{\mathrm{CO}_{2}}^{b} d_{\mathrm{CH}_{4}}^{3}=0.05$. Analytic formula of the exterior potential and surface mesh approach are used.

Finally, the excess adsorption quantities $\Gamma_{i}$ for each gas $\left(i=\mathrm{CH}_{4}, \mathrm{CO}_{2}\right)$ are defined as follows

$$
\Gamma_{i}=\frac{1}{\mathrm{~V}_{\text {pore }}} \int_{\mathrm{V}_{\text {pore }}}\left(\rho_{i}(\mathbf{r})-\rho_{i}^{b}\right) \mathrm{d} \mathbf{r}
$$

Figure 7 displays the excess adsorption isotherm of the gas mixture as function of the $\mathrm{CO}_{2}$ bulk density for a fixed $\mathrm{CH}_{4}$ bulk density. The adsorbed amount of $\mathrm{CO}_{2}$ increases more significantly with $\rho_{\mathrm{CO}_{2}}^{b}$ at low bulk density. On the other hand, the $\mathrm{CH}_{4}$ is withdrawn from the pore when increasing the $\mathrm{CO}_{2}$ bulk density due to the higher adsorption potential for $\mathrm{CO}_{2}$ than for $\mathrm{CH}_{4}$ as the 
Lennard-Jones exterior potential has higher values for $\mathrm{CO}_{2}$ than for $\mathrm{CH}_{4}$.

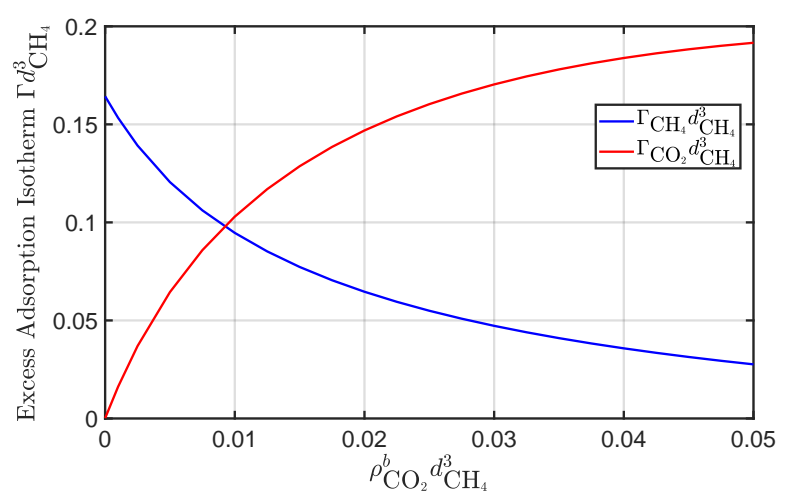

Figure 7: Normalized excess adsorption $\Gamma_{i} d_{\mathrm{CH}_{4}}^{3}$ versus $\mathrm{CO}_{2}$ bulk density with fixed $\mathrm{CH}_{4}$ bulk density $\rho_{\mathrm{CH}_{4}}^{b} d_{\mathrm{CH}_{4}}^{3}=0.05$.

\subsection{Solvation force calculation}

5.3.1. Analysis of the solvation force behavior The objective of this section is to characterize the solvation force by carrying out simulations for a single gas component $\left(\mathrm{CO}_{2}\right)$ in a spherical pore of radius $R_{p}=2.5 d_{\mathrm{CO}_{2}}$. The normalized bulk density $\rho_{\mathrm{CO}_{2}} d_{\mathrm{CO}_{2}}^{3}$ varies from 0 to 0.15 at a fixed temperature $T=333 \mathrm{~K}$, corresponding to a bulk pressure from 0 to $8 \mathrm{MPa}$. In this spherical configuration, the solvation force is a uniform pressure at the solid surface. Figure 8 plots the solvation force according to the bulk pressure. The solvation force is negative, acting as an attractive force between the fluid and the solid surface. Moreover, the magnitude of the force is much higher than the bulk pressure. At low bulk pressure, the magnitude of the solvation force increases quickly with an increase in bulk pressure (point A on figure 8) reaching a maximum value at a transition point (point B). For higher bulk pressure, the magnitude of the solvation force decreases smoothly with the bulk pressure (point $\mathrm{C}$ ). This behavior is explained by the molecules distribution in the pore and the derivative of exterior potential, keeping in mind that the integral of their product gives the solvation force (see [9] for details in the case of a spherical pore). In figures 9 , the derivative of the exterior potential and the gas density profiles corresponding to the three points considered in figure 8 for low, transition and high bulk pressures, are plotted. The normalized derivative of the exterior potential $-\beta d V^{e x t} / \mathrm{d} R_{p} d_{\mathrm{CO}_{2}}$ reaches high positive values in a thin zone adjacent to the exclusion zone where the interaction with the wall is repulsive. Far 
from the wall, this derivative is negative yielding an attractive force between the molecules and the solid surface. At low bulk pressure, the molecules in the pore have a tendency to be located in the attractive area so that increasing the bulk density leads to an increase in magnitude of the attractive force (or solvation force). At high values of the bulk pressure, by progressively increasing the pressure, more and more gas molecules are localized in the repulsive zone near the wall, thus reducing the magnitude of the solvation force (see figure $9 b$ ).

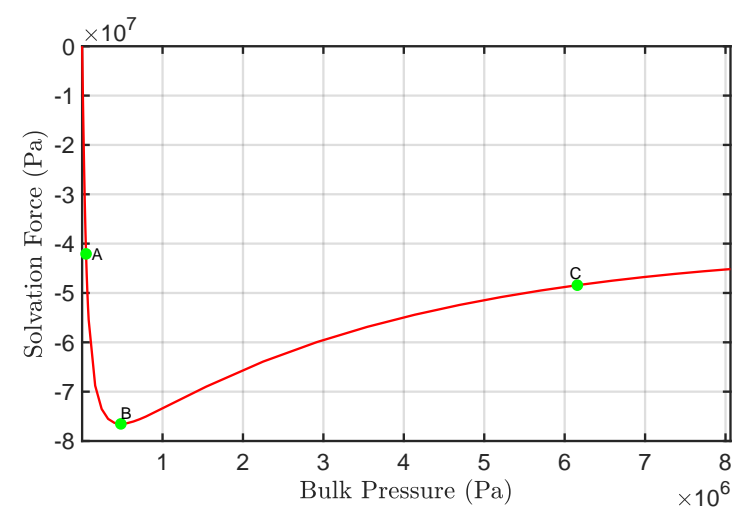

Figure 8: Solvation force versus $\mathrm{CO}_{2}$ bulk pressure in a spherical pore of radius $R_{p}=2.5 d$.

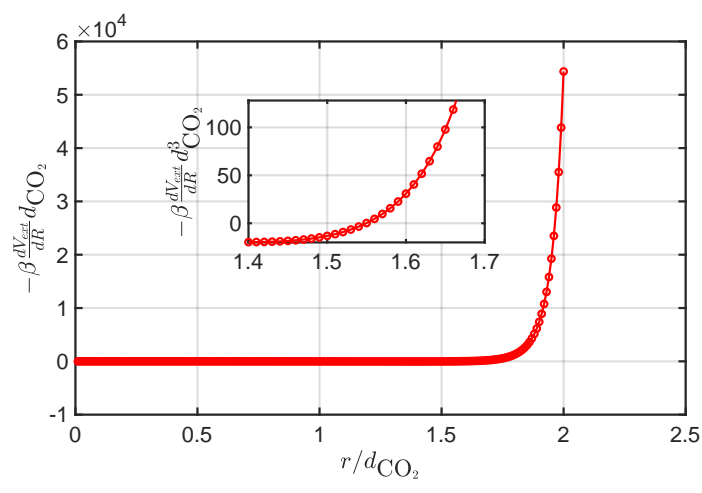

(a)

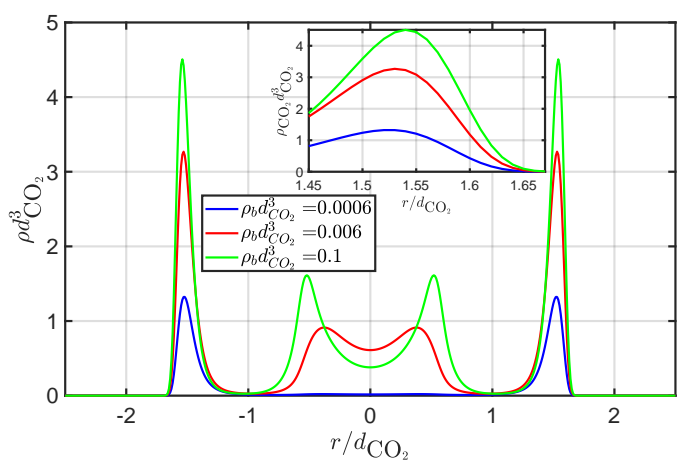

(b)

Figure 9: (a) Derivative of the exterior potential (b) Gas density profiles corresponding to three points for low (A), transition (B) and high (C) bulk pressures presented in figure 8 .

5.3.2. Ellipsoidal Cavity The solvation force is now computed at the surface of an oblate spheroidal pore of dimensions $a=b=4.5 d_{\mathrm{CH}_{4}}$ and for three value of $c=[3.5,4,4.5] d_{\mathrm{CH}_{4}}$. It 
should be noted that, unlike the spherical pore, in an ellipsoidal geometry the solvation force has normal and tangential components and the distribution of the solvation force at the solid surface is nonuniform. For an oblate spheroid, the solvation force profile can be reduced to 1D-presentation when projected on the $z$ axis. Figure 10(a) shows the normal component of solvation force for $0 \leq z \leq c$ where $z=0$ and $z=c$ correspond to the two extreme points. The normal force is negative and much higher in absolute value than the bulk pressure $P_{b}=8.31 \mathrm{MPa}$. For small value of $c / a$, this component varies considerably with respect to the position on the surface, tending to be uniform when $c / a \rightarrow 1$. Figure 10(b) displays the magnitude of the tangential component $\Pi^{\tau}$ according to $z$. We can observe that the tangential component is much smaller than the normal one and can therefore be neglected in the calculation of the solvation force. In addition, for a spherical cavity, the tangential component vanishes as expected.

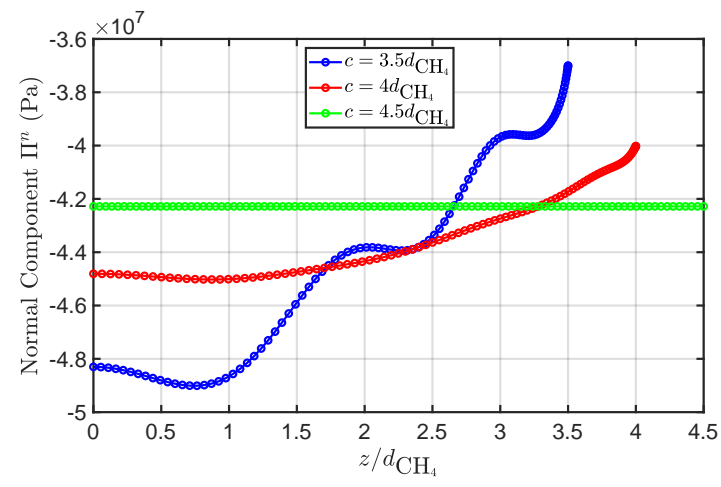

(a)

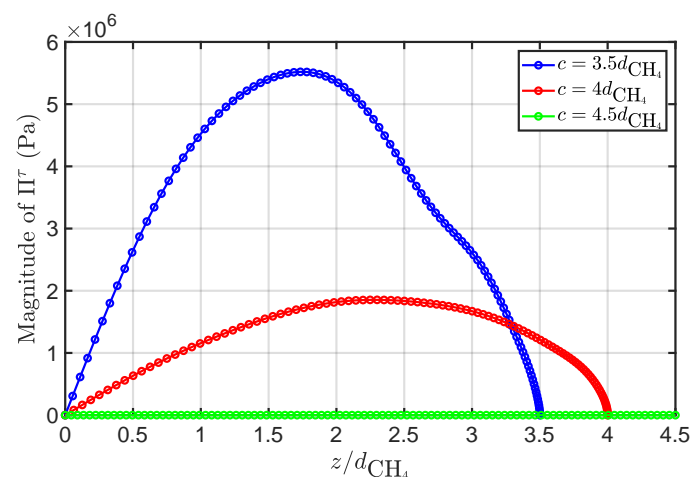

(b)

Figure 10: (a) Normal (b) and tangential components of the solvation force according to the axis $z$ in an oblate spheroidal pore, for $a=b=4.5 d_{\mathrm{CH}_{4}}, c=[3.5,4.0,4.5] d_{\mathrm{CH}_{4}}$ and bulk densities $\rho_{\mathrm{CH}_{4}}^{b} d_{\mathrm{CH}_{4}}^{3}=\rho_{\mathrm{CO}_{2}}^{b} d_{\mathrm{CH}_{4}}^{3}=0.05$ corresponding to a bulk pressure $P_{b}=8.31 \mathrm{MPa}$.

It should be noticed that our model is not restricted to the case of an ellipsoidal pore but can be generalized to any complex pore structure. As the magnitude of the solvation force can be very large and is different of the bulk pressure, this force has to be taken into account in the mechanical modeling. 


\section{Conclusion}

A new numerical method for solving the FMT equations applied to a Lennard-Jones fluid mixture in a nanopore of arbitrary shape has been developed in this work. The voxel discretization and the Fast Fourier Transformation allow solving efficiently the convolution terms using the analytic formula of the weight functions. The method has a tremendous advantage of being able to compute the sorbed fluid densities in three dimensions for any pore structure.

Once the fluid densities distribution in the pore is known, the solvation force, i.e. the force exerted by the adsorbed gas molecules on the solid surface, can be calculated from a novel formulation based on two different approaches for any type of geometry. A precise analysis of the solvation force behavior has been provided with particular reference to the case of $\mathrm{CH}_{4}$ and $\mathrm{CO}_{2}$ mixture adsorbed in nanopores of coal seam which is encountered in the enhanced coalbed methane recovery. It has been shown that this is an attractive force which depends on the fluid densities and the derivative of the exterior potential. For complex geometries as an ellipsoidal pore, the solvation force is composed of a normal part, which causes the swelling/shrinkage of the pore, and a tangential component which is much smaller than the normal one.

\section{Acknowledgements}

The authors would like to thank the EXPLOR center of the University of Lorraine for providing the computer resources necessary to perform the numerical simulations. Q.D. Ha also thanks the "Grand Est" Région for the research grant allowing him to carry out his $\mathrm{PhD}$ thesis.

\section{Data availability}

The data that support the findings of this study are available from the corresponding author upon reasonable request.

\section{References}

[1] C. H. Sondergeld, K. E. Newsham, M. C. Rice, C. S. Rai, Petrophysical considerations in evaluating and producing shale gas resources. society of petroleum engineers, Society of Petroleum Engineers, SPE Unconventional Gas Conference, Pennsylvania, USA 152 (2010) 10864-10868.

[2] J. Seidle, Fundamentals of Coalbed Methane Reservoir Engineering, PennWell Corp, 2011. 
[3] T. D. Le, M. A. Murad, P. A. Pereira, C. Boutin, Bridging between macroscopic behavior of shale gas reservoirs and confined fluids in nanopores, Computational Geosciences 20 (2016) 751-771.

[4] T. D. Le, M. A. Murad, A new multiscale model for flow and transport in unconventional shale oil reservoirs, Applied Mathematical Modelling 64 (2018) 453-479.

[5] P. I. Ravikovitch, A. V. Neimark, Density functional theory model of adsorption deformation, Langmuir 22 (2006) 10864-10868.

[6] S. Nikoosokhan, M. Vandamme, P. Dangla, A poromechanical model for coal seams injected with carbon dioxide: From an isotherm of adsorption to a swelling of the reservoir, Oil Gas Sci. Technol. Rev. IFP Energ. Nouvelles 67 (2012) 777-786.

[7] T. D. Le, Q. D. Ha, I. Panfilov, M. Moyne, Multiscale model for flow and transport in $\mathrm{co}_{2}$-enhanced coalbed methane recovery incorporating gas mixture adsorption effects, Advances in Water Resources 144 (2020) 47-60.

[8] G. Pijaudier-Cabot, R. Vermodel, C. Miqueu, B. Mendiboure, Revisiting poromechanics in the context of microporous materials, Comptes Rendus Mecanique 339 (2011) 770-778.

[9] T. D. Le, M. Moyne, M. A. Murad, I. Panfilov, A three-scale poromechanical model for swelling porous media incorporating solvation forces: Application to enhanced coalbed methane recovery, Mechanics of Materials 131 (2019) 47-60.

[10] R. Evans, The nature of the liquid-vapour interface and other topics in the statistical mechanics of non-uniform, classical fluids, Advances in Physics 28 (1979) 143-200.

[11] Y. Rosenfeld, Free-energy model for the inhomogeneous hard-sphere fluid mixture and density-functional theory of freezing, Physical Review Letters 63 (9) (1989) 980-983.

[12] Y. Rosenfeld, Free-energy model for the inhomogeneous hard-sphere fluid mixture: triplet and higher-order direct correlation functions in dense fluids, The Journal of Chemical Physics 92 (11) (1990) 6818-6832.

[13] J. K. Percus, G. J. Yevick, Analysis of classical statistical mechanics by means of collective coordinates, Physical Review 110 (1958) 1-13.

[14] G. A. Mansoori, N. F. Carnahan, K. E. Starling, T. W. Leland, Equilibrium thermodynamic properties of the mixture of hard spheres, Comptes Rendus Mecanique 54 (1971) 1523.

[15] R. Roth, Fundamental measure theory for hard-sphere mixtures: a review, J. Phys.: Condens. Matter 22 (2010) 063102.

[16] E. Kierlik, M. L. Rosinberg, Density-Functional Theory for inhomogeneous fluids: adsorption of binarymixtures, Physical Review A 44 (8) (1991) 5025-5037.

[17] A. Gonzalez, J. A. White, F. L. Roman, S. Velasco, Density functional theory of fluids in nanopores: analysis of the fundamental measures theory in extreme dimensional-crossover situations, The Journal of Chemical Physics 125 (2006) 64703.

[18] P. Tarazona, Density functional for hard sphere crystals: A fundamental measure approach, Phys. Rev. Lett. 84 (2000) 694.

[19] T. Bernet, E. A. Muller, G. Jackson, A tensorial fundamental measure density functional theory for the description of adsorption in substrates of arbitrary three dimensional geometry, The Journal of Chemical Physics 152 
(2020) 10864-10868.

[20] J.-P. Hansen, I. R. McDonald, Theory of Simple Liquids (Fourth Edition), Elsevier, 2013.

[21] J. D. Weeks, D. Chandler, H. C. Andersen, Role of repulsive forces in determining equilibrium structure of simple liquids, The Journal of Chemical Physics 54 (12) (1971) 5237.

[22] D. Bratko, L. Blum, M. S. Wertheim, Structure of hard sphere fluids in narrow cylindrical pores, The Journal of Chemical Physics 90 (1989) 2752-2757.

[23] S. C. Kim, S. H. Suh, C. H. Lee, Binary hard-sphere mixture within spherical pore, Journal of the Korean Physical Society 35 (1999) 350-354.

[24] D. D. Do, H. D. Do, Pore characterization of carbonaceous materials by DFT and GCMC simulations: a review, Adsorption Science and Technology 21 (2003) 389-423.

[25] U. Sievers, S. Schulz, An equation of state for methane in the form of Bender's equation for temperatures between $91 \mathrm{~K}$ and $625 \mathrm{~K}$ and pressures up to 500 bar, Fluid Phase Equilibria 5 (1) (1980) 35 - 54.

[26] R. Span, W. Wagner, A new equation of state for carbon dioxide covering the fluid region from the triple- point temperature to $1100 \mathrm{~K}$ at pressures up to $800 \mathrm{MPa}$, Journal of Physical and Chemical Reference Data 25 (6) (1996) $1509-1596$.

\section{Appendix A. Exterior potential}

\section{Appendix A.1. Spherical cavity}

In the case of a cavity of radius $R$, the potential (28) can be rewritten in the form

$$
V_{i}^{e x t}(x, y, z)=4 \varepsilon_{s i} \frac{N_{C}}{4 \pi R^{2}} \int_{S}\left[\left(\frac{\sigma_{s i}}{s}\right)^{12}-\left(\frac{\sigma_{s i}}{s}\right)^{6}\right] \mathrm{d} S
$$

where $N_{C}=\rho_{s} 4 \pi R^{2} \Delta$ is the solid atom number located at the solid/fluid interface. Consider the spherical coordinates $(r, \theta, \varphi)$

$$
\left\{\begin{aligned}
x & =r \sin \theta \cos \varphi & & 0<r<R \\
y & =r \sin \theta \sin \varphi & & 0<\theta<\pi \\
z & =r \cos \theta & & 0<\varphi<2 \pi
\end{aligned}\right.
$$

The volume and surface element $\mathrm{d} V$ and $\mathrm{d} S$ are given by

$$
\begin{aligned}
& \mathrm{d} V=r^{2} \mathrm{~d} r \sin \theta \mathrm{d} \theta \mathrm{d} \varphi \\
& \mathrm{d} S=R^{2} \sin \theta_{S} \mathrm{~d} \theta_{S} \mathrm{~d} \varphi_{S}
\end{aligned}
$$

The distance $s$ between a point on the surface of coordinates $\left(R, \theta_{S}, \varphi_{S}\right)$ and a molecule of the fluid at the point of coordinates $(r, \theta, \varphi)$ is given by

$$
s^{2}=r^{2}-2 r R\left[\sin \theta_{S} \sin \theta \cos \left(\varphi_{S}-\varphi\right)+\cos \theta_{S} \cos \theta\right]+R^{2}
$$


The total exterior potential $V_{i}^{\text {ext }}$ representing the force of the surface $s$ exerting on a molecule at the position $(r, \theta, \varphi)$ is written as

$$
V_{i}^{e x t}(r, \theta, \varphi ; R)=R^{2} \int_{\theta_{S}=0}^{\pi} \sin \theta_{S} \mathrm{~d} \theta_{S} \int_{\varphi_{S}=0}^{2 \pi} \mathrm{d} \varphi_{S} v_{i}(s)
$$

In the spherical geometry, $V_{i}^{\text {ext }}(r ; R)$ depends only on $r$ and is independent of $\theta$ and $\varphi$. To simplify, the calculation will be carried out for $\theta=0$ and $\varphi=0$. It yields

$$
s^{2}=r^{2}-2 r R \cos \theta_{S}+R^{2}
$$

leading to

$$
V_{i}^{e x t}(r ; R)=2 \varepsilon_{s i} N_{c} \int_{\theta_{S}=0}^{\pi} \sin \theta_{S} \mathrm{~d} \theta_{S}\left[\left(\frac{\sigma_{s i}}{s}\right)^{12}-\left(\frac{\sigma_{s i}}{s}\right)^{6}\right]
$$

\section{Appendix A.2. Ellipsoidal cavity}

We now consider a more complex geometry of an ellipsoidal cavity with three semi-axes $a, b$ and $c$ which satisfy the following relation in Cartesian coordinate system

$$
\left(\frac{x}{a}\right)^{2}+\left(\frac{y}{b}\right)^{2}+\left(\frac{z}{c}\right)^{2}=1
$$

An alternative way to define the surface is to use the parametric equations given by

$$
\mathbf{X}=\left\{\begin{array}{l}
x=a \sin \theta_{S} \cos \varphi_{S} \\
y=b \sin \theta_{S} \sin \varphi_{S} \\
z=c \cos \theta_{S}
\end{array}\right.
$$

Two tangent vectors to the ellipsoid surface are obtained by differentiating the previous expressions with respect to $\theta_{S}$ and $\varphi_{S}$

$$
\begin{aligned}
& \frac{\partial \mathbf{X}}{\partial \theta_{S}}=\left(a \cos \theta_{S} \cos \varphi_{S}, b \cos \theta_{S} \sin \varphi_{S},-c \sin \theta_{S}\right) \\
& \frac{\partial \mathbf{X}}{\partial \varphi_{S}}=\left(-a \sin \theta_{S} \sin \varphi_{S}, b \sin \theta_{S} \cos \varphi_{S}, 0\right)
\end{aligned}
$$

The surface element $\mathrm{d} S$ is then obtained as

$$
\begin{aligned}
\mathrm{d} S & =\left\|\frac{\partial \mathbf{X}}{\partial \theta_{S}} \wedge \frac{\partial \mathbf{X}}{\partial \varphi_{S}}\right\| \mathrm{d} \theta_{S} \mathrm{~d} \varphi_{S} \\
& =\sqrt{a^{2} b^{2} \cos ^{2} \theta_{S}+\left(a^{2} \sin ^{2} \varphi_{S}+b^{2} \cos ^{2} \varphi_{S}\right) c^{2} \sin ^{2} \theta_{S}} \times \sin \theta_{S} \mathrm{~d} \theta_{S} \mathrm{~d} \varphi_{S}
\end{aligned}
$$

and the external potential $V_{i}^{\text {ext }}(\mathbf{r})$ is immediately deduced from

$$
V_{i}^{e x t}(\mathbf{r})=4 \varepsilon_{s i} \rho_{s} \Delta \int\left[\left(\frac{\sigma_{s i}}{s}\right)^{12}-\left(\frac{\sigma_{s i}}{s}\right)^{6}\right] \mathrm{d} S
$$


Note that the unit normal vector of the surface is given by

$$
\begin{aligned}
\mathbf{n}=\frac{\frac{\partial \mathbf{X}}{\partial \theta_{S}} \wedge \frac{\partial \mathbf{X}}{\partial \varphi_{S}}}{\left\|\frac{\partial \mathbf{X}}{\partial \theta_{S}} \wedge \frac{\partial \mathbf{X}}{\partial \varphi_{S}}\right\|} & =\frac{1}{\sqrt{a^{2} b^{2} \cos ^{2} \theta_{S}+\left(a^{2} \sin ^{2} \varphi_{S}+b^{2} \cos ^{2} \varphi_{S}\right) c^{2} \sin ^{2} \theta_{S}}} \\
& \times\left(\begin{array}{l}
b c \sin \theta_{S} \cos \varphi_{S} \\
a c \sin \theta_{S} \sin \varphi_{S} \\
a b \cos \theta_{S}
\end{array}\right)
\end{aligned}
$$

\section{Appendix B. Analytic Fourier transform of the weight functions}

The Fourier transform $\widehat{V}(\mathbf{k})$ in three-dimensional space of a function $V(\mathbf{r})$ is defined by

$$
\widehat{f}(\boldsymbol{k})=\iiint f(\boldsymbol{r}) \exp (-i \boldsymbol{k} \cdot \boldsymbol{r}) d \boldsymbol{r}
$$

where $\boldsymbol{r}$ and $\boldsymbol{k}$ denote the coordinate vectors in real and Fourier spaces respectively.

When $f$ is only function of the distance, its Fourier transform depending on $k=|\mathbf{k}|$ is given as

$$
\widehat{f}(k)=4 \pi \int_{0}^{\infty} f(r) \frac{r \sin (k r)}{k} d r
$$

By using the above relation, the Heaviside step function representing the weight function $\omega_{3}^{i}$ in the Fourier space is written as

$$
\widehat{H}^{i}(\mathbf{k})=4 \pi \int_{0}^{R_{i}} \frac{r \sin (k r)}{k} d r=4 \pi \frac{\sin \left(k R_{i}\right)-k R_{i} \cos \left(k R_{i}\right)}{k^{3}}
$$

It is straightforward to obtain the Fourier transform of the weight functions, which depend only of the distance $r=|\mathbf{r}|$ :

$$
\left\{\begin{array}{l}
\widehat{\omega}_{3}^{i}(\mathbf{k})=4 \pi \frac{\sin \left(k R_{i}\right)-k R_{i} \cos \left(k R_{i}\right)}{k^{3}} \\
\widehat{\omega}_{2}^{i}(\mathbf{k})=4 \pi R_{i} \frac{\sin \left(k R_{i}\right)}{k} \\
\widehat{\omega}_{1}^{i}(\mathbf{k})=\frac{\sin \left(k R_{i}\right)}{k} \\
\widehat{\omega}_{0}^{i}(\mathbf{k})=\frac{\sin \left(k R_{i}\right)}{k R_{i}} \\
\widehat{\omega}_{2}^{i}(\mathbf{k})=-\nabla \widehat{\omega}_{3}^{i}=-i \mathbf{k} \widehat{\omega}_{3}^{i}=-4 \pi i \frac{\sin \left(k R_{i}\right)-k R_{i} \cos \left(k R_{i}\right)}{k^{3}} \mathbf{k} \\
\widehat{\omega}_{1}^{i}(\mathbf{k})=\frac{\widehat{\omega}_{2}^{i}}{4 \pi R_{i}}=-i \frac{\sin \left(k R_{i}\right)-k R_{i} \cos \left(k R_{i}\right)}{k^{3} R_{i}} \mathbf{k}
\end{array}\right.
$$

It should be noticed that, the weight functions in the Fourier space are analytic, depending only on the Fourier parameter $\boldsymbol{k}$ and molecule radius $R_{i}$. 